\title{
MOTILITY
}

\section{Baclofen effective for rumination and supragastric belching in a pilot study}

Rumination and supragastric belching can reduce a patient's quality of life, yet the current standard treatment-behavioral therapy-is not always effective. A pilot study has now shown that baclofen can improve the symptoms of these disorders.

Baclofen, an agonist of $\gamma$-aminobutyric acid B receptor, increases lower esophageal sphincter (LES) pressure and decreases the swallowing rate; low LES pressure postprandially and a high swallowing rate are characteristic of rumination and supragastric belching. As baclofen can improve these symptoms in patients with GERD, it could be effective in patients with rumination or supragastric belching.

The pilot study included 12 patients who had these two disorders. At baseline, high-resolution manometry-impedance recordings (which measure flow events) were taken for $30 \mathrm{~min}$, patients then ate a meal and recordings were taken for a further $60 \mathrm{~min}$. The patients recorded their symptoms with an event marker. The process was repeated after the patients had received $10 \mathrm{mg}$ of baclofen three times a day for 1 week.

The number of flow events and symptoms were significantly reduced following treatment, and an increase in LES pressure was noted. Patients reported only minor adverse events.

This pilot study indicates that baclofen is safe and effective for patients with rumination or supragastric belching; however, a placebo-controlled trial in a large cohort of patients is necessary to confirm these findings.

\section{Claire Greenhill}

Original article Blondeau, K. et al. Baclofen improves
symptoms and reduces postprandial flow events in patients
with rumination and supragastric belching.
Clin. Gastroenterol. Hepatol. doi:10.1016/
j.cgh.2011.10.042

